\title{
EVALUACIÓN DE LA ESTRUCTURA POROSA DE LOS MATERIALES MCM-22, MCM-36 E ITQ-2 EMPLEANDO EL TEST CATALITICO DEL N-DECANO
}

\author{
Sibele B. C. Pergher* \\ Departamento de Química, Universidade Regional Integrada do Alto Uruguai e das Missões, Campus Erechim, Av. Sete de Setembro, \\ 1621, 99700-000 Erechim - RS \\ Avelino Corma y Vicente Fornés \\ Instituto de Tecnología Química, Universidad Politécnica de Valencia, Avda. de los Naranjos , s/n , 46022 Valencia - España
}

Recebido em 26/11/02; aceito em 14/3/03

\begin{abstract}
EVALUATION OF THE PORE STRUCTURE OF MCM-22, MCM-36 AND ITQ-2 MATERIALS USING THE N-DECANE CATALYTIC TEST. The void structure of zeolites MCM-22, MCM-36 and ITQ-2 were discussed on the bases of catalytic reaction tests. The hydromerization of n-decane on bifunctional Pt/Zeolite Catalysts have been used as model reactions. Beta and ZSM-5 zeolites were used for comparison. It is concluded that all materials show features of 10MR zeolites and have also pores bigger than $12 \mathrm{MR}$ in this order MCM-22<MCM-36<ITQ-2.
\end{abstract}

Keywords: n-decane test; zeolites; ITQ-2.

\section{INTRODUCCIÓN}

Diversas reacciones modelos han sido empleadas para determinar la topología porosa en zeolitas de estructura desconocida. Particularmente, el test del n-decano nos ofrece informaciones valiosas respecto a características de la geometría porosa, tales como: número de tetraedros que limitan las ventanas de los canales (poros) y la presencia de intersección de canales o cavidades. Martens et al. en $1984^{1}$ desarollaron este test y, a través de una análisis de distribución de los productos de la reacción, determinaron cinco critérios independientes que permiten estimar la estructura del catalizador:

1) Distribución relativa de isómeros monorramificados contra birramificados en conversión máxima a isómeros.

2) Contribución relativa de isómeros etiloctano en la mezcla de isómeros monorramificados en bajas conversiones a isómeros.

3) Relación 3-etiloctano/4-etiloctano.

4) Distribución relativa de isómeros individuales monorramificados (2-metilnonano/5-metilnonano) en bajas conversiones a isómeros.

5) Rendimiento absoluto de isopentano en productos en bajas conversiones de hidrocraqueo.

El método se basa en la comparación de seletividades obtenidas en zeolitas desconocidas con las de zeolitas de referencia (de estructura y microporosidad conocidas).

El método fue empleado para dilucidar la estructura del material MCM-222,3. En ambos trabajos se llegó a la conclusión de que la MCM-22 muestra un comportamiento característico de materiales con poros tanto de 10MR como de 12MR, dependiendo del criterio utilizado. Esto se explica asumiendose la presencia de ambos tipos de canales. Posteriormente a estos estudios, la estructura fue "revelada", la misma presenta dos sistemas independientes de poros: uno formado por cavidades de 12MR (18 x $7 \AA$ ) conectadas por aberturas de 10MR; y otro, definido por un sistema de canales sinusoidales de $10 \mathrm{MR}^{4,5}$. Realmente, el material posee poros con dimensiones de 10MR y 12MR (Figura 1).

*e-mail: pergher@uricer.edu.br

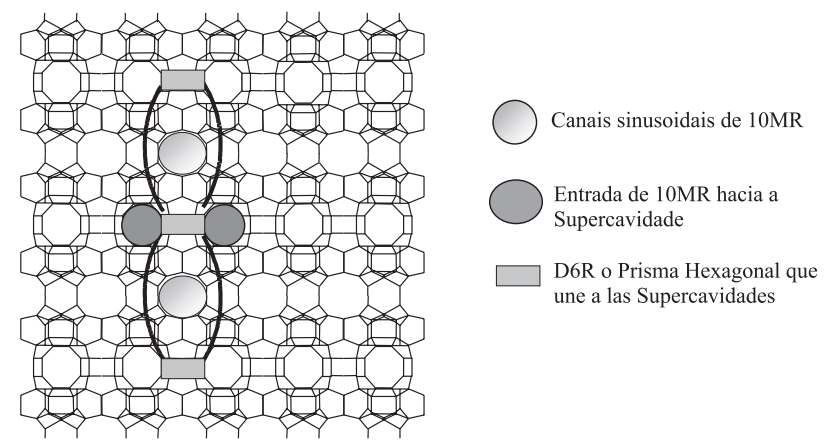

Figura 1. Estructura de la $M C M-22^{8}$

Partiendo del precursor zeolitico de la MCM-22, que es un precursor laminar, se pueden obtener otros materiales: MCM-36 ${ }^{6,7}$ e ITQ$2^{8-12}$.

La diferencia de las estructuras se basa en disposición de las láminas. En el caso de la MCM-22, las mismas están condensadas formando los dos sistemas de poros yá descriptos; en la MCM-36, las láminas están separadas por pilares de Si; y en la ITQ-2, las láminas están desordenadas al azar (Figura 2).

El objetivo de este trabajo fue evaluar la estructura porosa de estos materiales empleando el test del n-decano.

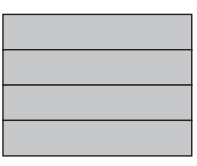

MCM-22

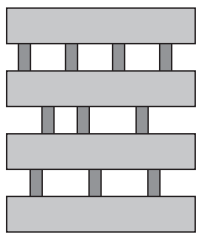

MCM-36

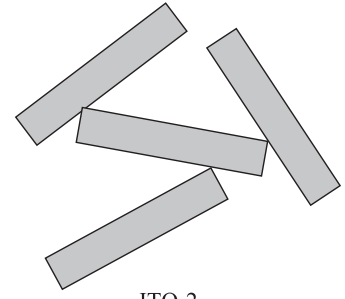

ITQ-2
Figura 2. Representación esquemática de la estructura de los materiales MCM-22, MCM-36 e ITQ-2 


\section{EXPERIMENTAL}

Los materiales MCM-22, MCM-36 e ITQ-2 fueron preparados conforme literatura ${ }^{4-15}$ y evaluados catalíticamente a través del test del n-decano.

La reacción de hidroisomerización del n-decano fue llevada a cabo en un sistema de reacción, donde hay dos líneas de alimentación: una de $\mathrm{H}_{2}$, que pasa por el saturador, que es para la reacción propiamente dicha; y otra que son de $\mathrm{H}_{2}$ y $\mathrm{N}_{2}$ para el pretratamiento de los catalizadores. La salida del reactor puede ser direccionada al cromatógrafo o a un colector y medidor de flujo. Las líneas que conectan el saturador con el reactor, y éste último con el cromatógrafo, están revestidas por mantas calentadoras a $80{ }^{\circ} \mathrm{C}$ y $180{ }^{\circ} \mathrm{C}$ respectivamente, para evitar la condensación de reactante y productos.

El saturador de vapor consta de una pieza de vidrio $(5,2 \mathrm{~cm}$ de diámetro y $21,5 \mathrm{~cm}$ de altura), que contiene el n-decano y por donde pasará el gas de arrastre $\left(\mathrm{H}_{2}\right)$. Esta pieza de vidrio está inmersa en un baño de silicona con agitación magnética y con la temperatura controlada por un termopar que regula el calentamiento. La temperatura se mantiene a $53{ }^{\circ} \mathrm{C}$, en la cual se obtiene una relación $\mathrm{H}_{2} / \mathrm{n}$-decano $=100$. El cálculo de la temperatura a la que se obtiene la relación $\mathrm{H}_{2} /$ n-decano $=100$ fue realizado utilizando la ecuación y los datos de ndecano, como se muestra a continuación:

$\ln \left(\mathrm{P}_{\mathrm{vP}} / \mathrm{P}_{\mathrm{c}}\right)=(1-\mathrm{x})^{-1}\left[\alpha \mathrm{x}+\beta \mathrm{x}^{1.5}+\gamma \mathrm{x}^{3}+\delta \mathrm{x}^{6}\right]$

siendo $\mathrm{x}=1-\left(\mathrm{T} / \mathrm{T}_{\mathrm{c}}\right)$

$\begin{array}{lll}\text { Para el n-decano }{ }^{16}: & \mathrm{T}_{\mathrm{c}}=617.7 \mathrm{~K} & \mathrm{P}_{\mathrm{c}}=21.2 \mathrm{bar} \\ & \alpha=-8.56523 & \beta=1.97756 \\ & \gamma=-5.81971 & \delta=-0.29982\end{array}$

El reactor es un tubo cilíndrico de cuarzo con un tubo fino interno donde se coloca el termopar para medir la temperatura del lecho catalítico, posee una placa porosa donde se colocará lana de cuarzo y lecho catalítico. El reactor de cuarzo $(2 \mathrm{~cm}$ de diámetro y $33 \mathrm{~cm}$ de altura) se encuentra dentro de un horno. Tanto el horno como las mantas calefactoras están controlados por un controlador de temperatura. Las respectivas temperaturas son medidas por termopares.

El "test" del n-decano es una reacción bifuncional donde participan los sitios metálicos y ácidos. Los catalizadores utilizados están en su forma ácida, la función metálica es obtenida a través del Platino (Pt) impregnado en estos materiales. Se utilizó $1 \%$ de Pt en peso $^{1}$.

La impregnación se llevó a cabo adicionándose una solución de $\mathrm{H}_{2} \mathrm{PtCl}_{6} / \mathrm{HCl}(0,2 \mathrm{~mol} / \mathrm{L})$ en el catalizador en una proporción de $3 \mathrm{ml}$ de solución por $1 \mathrm{~g}$ de catalizador. La cantidad de Pt adicionada fue del $1 \%$ en peso. La suspensión fue secada en rotavapor y posteriormente en estufa a $100{ }^{\circ} \mathrm{C}$. Después de seco, el material impregnado es calcinado en mufla a $500{ }^{\circ} \mathrm{C}$ durante $3 \mathrm{~h}$. Después de calcinado, el catalizador es pastillado (5Ton) y tamizado. Se utiliza la fracción de $0,59 \mathrm{~mm}$ a $0,84 \mathrm{~mm}$. Se mezcla $0,5 \mathrm{~g}$ del catalizador con $1,5 \mathrm{~g}$ de $\mathrm{SiO}_{2}(0,25$ a $0,42 \mathrm{~mm})$ lo que constituirá el lecho catalítico. Antes de utilizar el catalizador en reacción, el mismo se debe activar en el propio reactor en presencia de $\mathrm{H}_{2}$ a $450{ }^{\circ} \mathrm{C}$ durante $2 \mathrm{~h}(300 \mathrm{ml} / \mathrm{min})$.

Una vez activado el catalizador, se inicia la reacción. Se alimenta el reactor con la corriente de gas que sale del saturador, $\mathrm{H}_{2} / \mathrm{n}$ decano $=100\left(a^{\circ} 5{ }^{\circ} \mathrm{C}\right)$. Se inicia con una temperatura de reacción de $130{ }^{\circ} \mathrm{C}$ y se aumenta de 10 en 10 grados, variando la conversión de 0 a $100 \%$. En cada temperatura se analizan los productos de reacción en un cromatógrafo de gases en línea con el sistema de reacción.

El cromatografo utilizado fue un Hewlett Packard modelo HP5890 serie II con un detector de ionización de llama (FID). La columna utilizada fue HP1 de $25 \mathrm{~m}$ x 0,2 mm x 0,33 $\mu \mathrm{m}$ (espesor del film) con metil silicona. El integrador fue el modelo HP3394. Las condiciones de operación fueron las siguientes: temperatura del inyector $=200{ }^{\circ} \mathrm{C}$; temperatura del detector $=250{ }^{\circ} \mathrm{C}$; temperatura de la columna $=30^{\circ} \mathrm{C}$ por $5 \mathrm{~min}$, se calienta $10^{\circ} \mathrm{C} / \mathrm{min}$ hasta $140{ }^{\circ} \mathrm{C} \mathrm{y}$ se mantiene por $5 \mathrm{~min}$; temperatura de la válvula de inyección = $175{ }^{\circ} \mathrm{C}$; presión de la columna (cabeza) $=10$ psi y los flujos del detector fueron: $\mathrm{H}_{2}=31.6 \mathrm{ml} / \mathrm{min}(1.7 \mathrm{bar})$; aire $=400 \mathrm{ml} / \mathrm{min}$ ( $3.1 \mathrm{bar}$ ) y makeup= $19.3 \mathrm{ml} / \mathrm{min}$.

\section{RESULTADOS Y DISCUSIÓN}

En primer lugar, se ha estudiado el "test" del n-decano sobre dos zeolitas de estructura conocida, comparando nuestros resultados con los obtenidos por Martens et al. ${ }^{1}$. Para tal, fueron empleados dos catalizadores zeolíticos comerciales con las características fisico-químicas presentadas en la Tabla 1 .

Tabla 1. Características de los catalizadores comerciales utilizados

\begin{tabular}{|c|c|c|c|c|}
\hline Catalizador & $\mathrm{Si} / \mathrm{Al}$ & $\begin{array}{c}\text { Área BET } \\
\left(\mathrm{m}^{2} / \mathrm{g}\right)\end{array}$ & $\begin{array}{c}\mathrm{Na}_{2} \mathrm{O} \\
\text { (\% \%eso) }\end{array}$ & Observaciones \\
\hline $\begin{array}{l}\text { ZSM-5 } \\
\text { CBV5020 }\end{array}$ & 26,5 & 423 & 0,02 & $\begin{array}{l}\text { tamaño de cristal } \\
1-3 \mu \mathrm{m} \text { poros } \\
\text { de } 10 \mathrm{MR}\end{array}$ \\
\hline $\begin{array}{l}\text { BETA } \\
\text { CP811 }\end{array}$ & 12,5 & 730 & 0,02 & poros de $12 \mathrm{MR}$ \\
\hline
\end{tabular}

Los productos de reacción fueron identificados por cromatografía gaseosa y espectometría de masas. Por cromatografía gaseosa, utilizando patrones de compuestos puros, se identificó la mayor parte de los productos hasta C9. La identificación de los isómeros C10 se llevó a cabo utilizando espectrometría de masas.

Los resultados catalíticos obtenidos sobre la zeolita Beta se presentan en la Figura 3. A partir de estos resultados se obtienen los criterios del "test" del n-decano (Figuras 4 a 7). Las zeolitas Beta y ZSM-5 fueron elegidas por el hecho de que la primera está formada por canales con anillos de 12 miembros, mientras que la segunda está formada por canales con anillos de 10 miembros.

Los resultados obtenidos en este trabajo son semejantes a los obtenidos por Martens et al. ${ }^{1}$, y en cualquier caso permiten llegar a las mismas conclusiones. Así pues, los resultados obtenidos con las zeolitas ZSM-5 y Beta servirán como referencia para discutir los obtenidos sobre la MCM-22, MCM-36 e ITQ-2. Por lo tanto, los resultados obtenidos con el "test" para los otros materiales serán comparados con los resultados de los materiales Beta y ZSM-5 obtenidos en las mismas condiciones de reacción.

Cuando se comparan los resultados obtenidos para la hidroisomerización-hidrocraqueo del n-decano sobre los materiales MCM-22, MCM-36 e ITQ-2, se observa (Figura 4) que el material ITQ-2 produce una relación mono/dirramificados más próxima a 0,5 ; a continuación le sigue la MCM-36 y, por último, la MCM-22. Estos resultados parecen indicar que la ITQ-2 posee poros más grandes que la MCM-36; y ésta, más grandes que la MCM-22. Sin embargo, a la hora de interpretar los resultados se debe considerar que en los tres materiales existe el sistema de poros formados por anillos de 10 miembros, lo cual se pone de manifiesto al considerar los resultados correspondientes al segundo criterio (Figura 5). Además, hay que considerar que las cavidades con diámetro de 12MR en la MCM-22 son accesibles sólo a través de ventanas de 10MR, mientras que en la MCM-36 lo son a través de poros formados entre los pilares, y en la ITQ-2 son totalmente accesibles. En consecuencia, se podría predecir 

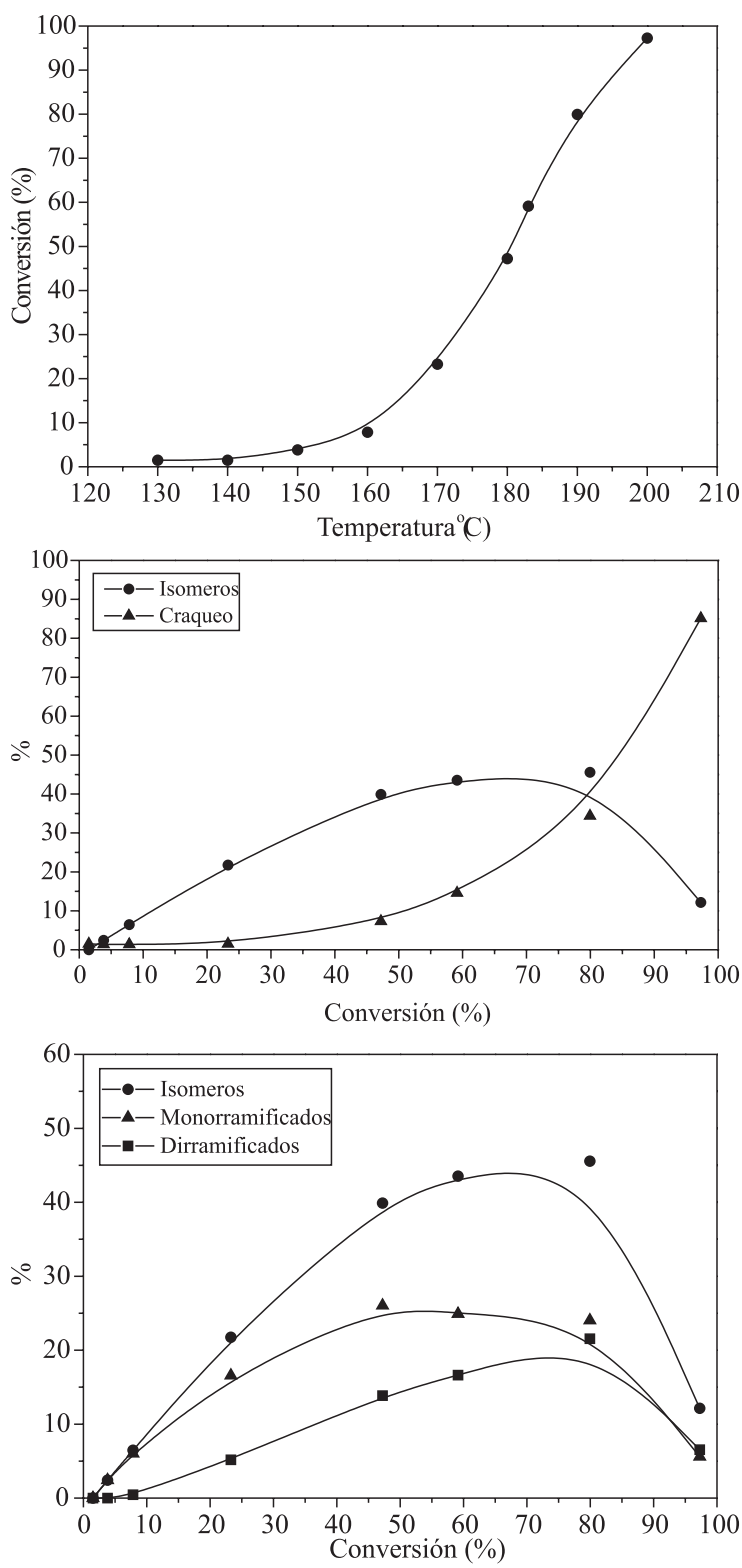

Figura 3. Resultados del "test" del n-decano para el material Beta. Conversión del n-decano a diferentes temperaturas. Selectividad a isómeros y a productos de craqueo

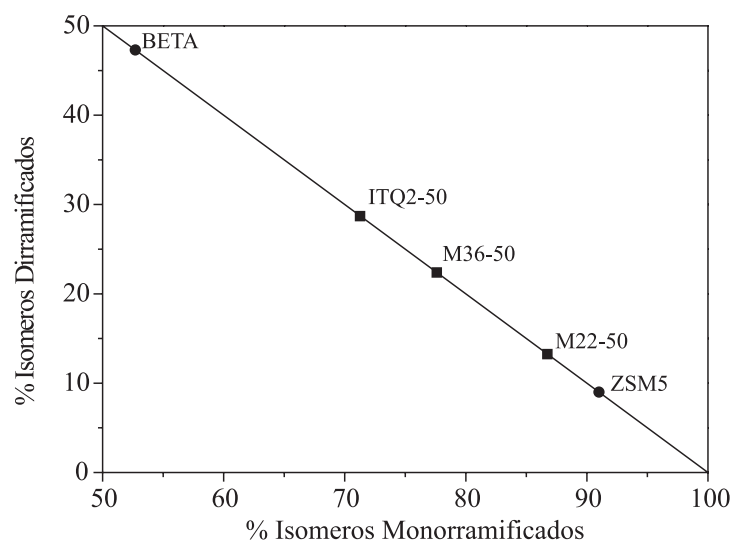

Figura 4. Distribución relativa de isómeros monorramificados contra dirramificados, obtenida al máximo de conversión a isómeros $\left(1^{\circ}\right.$ Criterio $)$ que tanto la MCM-36 como la ITQ-2 darán una relación mono/ dirramificado más alta que la MCM-22. Una posible desaluminización preferente o bloqueo de las copas de $12 \mathrm{MR}$, resaltaría la influencia de los canales de 10MR.

Este efecto también influirá en el segundo criterio, como se observa en la Figura 5, donde se ve únicamente la contribución de los canales de 10MR sinusoidales.

En la Figura 6, y de acuerdo con el cuarto criterio del "test" de ndecano, se pone de manifiesto que las cavidades formadas por poros de 12 MR juegan un papel fundamental en este caso, resultando un comportamiento típico de estructuras de poro grande. Finalmente, cuando se aplica el $5^{\circ}$ criterio (Figura 7) se observa que tanto la ITQ2 como la MCM-22 producen elevadas cantidades de iC5, ya que las

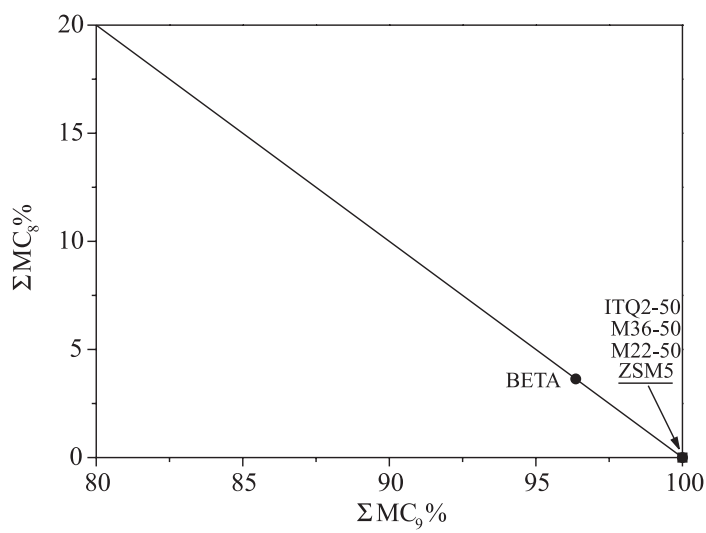

Figura 5. Rendimiento de isómeros etiloctano (EC8) contra metilnonano (MC9), obtenido a $5 \%$ de conversión a isómeros $\left(2^{\circ}\right.$ Criterio $)$

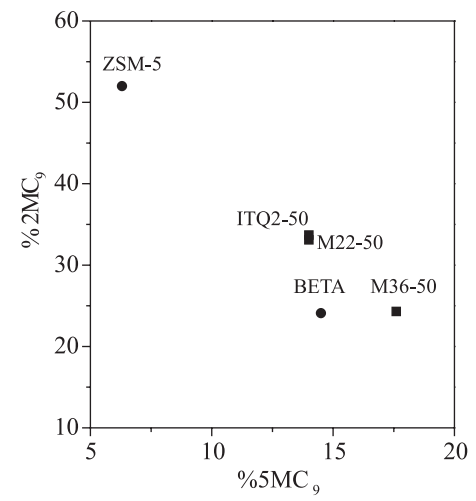

Figura 6. Concentración relativa de $2 M C 9$ contra $5 M C 9$ en los isómeros monometilramificados a 5\% de conversión a isómeros $\left(4^{\circ}\right.$ Criterio)

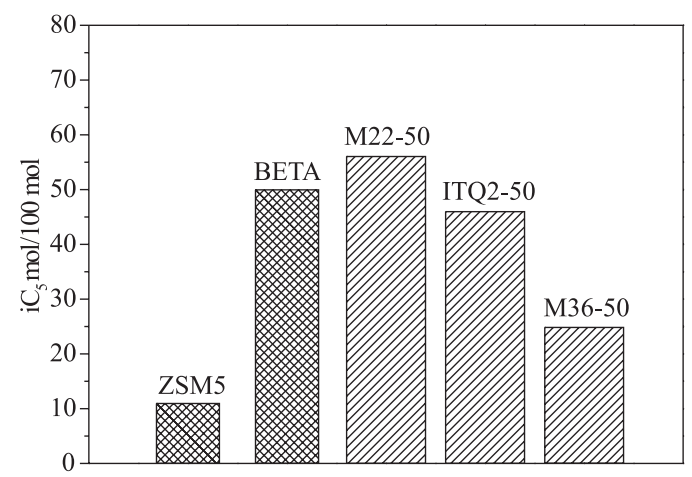

Figura 7. Rendimiento absoluto de iC5 formado a $5 \%$ de conversión al craqueo $\left(5^{\circ}\right.$ Criterio $)$ 
moléculas con varias ramificaciones, formadas en las cavidades, pueden craquear dando iC5. En el caso de la MCM-36, su baja aci$\mathrm{dez}^{7}$ produce poco hidrocraqueo.

\section{CONCLUSIÓN}

Por la evaluación de porosidad de los materiales MCM-22, MCM36 e ITQ-2, a través del método catalítico del n-decano, se concluye en que todos ellos presentan un comportamiento característico de materiales formados por 10MR, lo que concuerda con la estructura descripta para láminas que poseen canales sinusoidales de 10MR. Las diferentes estructuras están definidas por la forma en que las láminas se encuentran ordenadas: En la MCM-22, están condensadas; en la MCM-36, separadas por pilares; y en la ITQ-2, totalmente desordenadas. De esta manera, en la MCM-22, las cavidades formadas por 12MR son accesibles por ventanas de 10MR; en la MCM36 , por mesoporos formados entre pilares; y en la ITQ-2; totalmente accesibles.

Por evaluación catalítica, se concluye también que la estructura de la ITQ-2 es la que presenta poros de mayor tamaño, seguida de la MCM-36 y, por último, la MCM-22. Características que están de acuerdo con la accesibilidad esperada.

\section{REFERENCIAS}

1. Martens, J. A.; Tielen, M.; Jacobs, P. A.; Weitkamp, J.; Zeolites 1984, 4, 98.

2. Corma, A.; Corell, C.; Llopis, F.; Martinéz, A.; Pérez-Pariente, J.; Appl. Catal., A 1994, 115, 121.

3. Souverijns, W.; Verrelst, W.; Vanbutsele, G.; Martens, J. A.; Jacobs, P. A.; J. Chem. Soc., Chem. Commun. 1994, 1671.

4. Rubin, M. K.; Chu, P.; US pat. 49543251990.

5. Leonowicz, M. E.; Lawton, J. A.; Lawton, S. L.; Rubin; M. K.; Science 1994, 264, 1910.

6. Kresge, C. T.; Roth, W. L.; Simons, K. G.; Vartuli, J. C.; Word pat. WO92/ 11934.

7. Pergher, S. B. C.; Corma, A.; Fornés,V.; Acta Scientiarium 2003, submetido.

8. Pergher, S. B. C.; Tese de Doutorado, Universidad Politécnica de Valencia, Espanha, 1997.

9. Corma, A.; Fornés, V.; Guil, J. M.; Martinez-Triguero, J.; Pergher, S. B. C.; Maessen, TH. L. M.; Buglass, J. G.; Microporous Mesoporous Mater. 2000, 38, 301 .

10. Corma, A.; Fornes, V.; Martinez-Triguero, J.; Pergher, S. B. C.; J. Catal. 1999, 186, 57.

11. Corma, A.; Fornes, V.; Pergher, S. B. C.; Maessen, TH. L. M.; Buglass, J. G.; Nature 1998, 396, 353.

12. Pergher, S. B. C.; Corma, A.; Fornes, V.; XVIII Simpósio Iberoamericano de Catálisis, Porlamar - Isla Margarita, Venezuela, 2002.

13. Corma, A.; Corell, C.; Perez-Pariente, J.; Zeolites 1995, 15, 2.

14. Corma, A.; Corell, C.; Fornés, V.; Kolodziejski, W.; Perez-Pariente, J.; Zeolites 1995, 15, 576.

15. Pergher, S. B. C.; Corma, A.; Fornés, V.; Quim. Nova, in press.

16. Reid, R. C.; Prausnitz, J. M.; Poling, B. E.; The Properties of Gases and Liquids, $4^{\circ}$ ed., Mc Graw-Hill. 